\title{
Some approximation properties of Urysohn type nonlinear operators
}

\author{
Harun Karsli
}

\begin{abstract}
The central issue of this paper is to continue the investigation of convergence properties of Urysohn type operators. By using Urysohn type operators we will extend the theory of interpolation to functionals and operators. In details, the present paper centers around Urysohn type nonlinear counterpart of the two dimensional Stancu operators defined on a triangle. We construct our nonlinear operators by defining a nonlinear forms of the kernel functions. Afterwards, we investigate the convergence problem for these operators.
\end{abstract}

Mathematics Subject Classification (2010): 41A25, 41A35, 47G10, 47H30.

Keywords: Urysohn integral operators, Stancu operator, two dimensional nonlinear Stancu operators, Urysohn type nonlinear Stancu operators.

\section{Introduction}

In functional analysis, the superposition problem is known as the problem of representing a function $f$ as the composition of "simpler and more easily calculated" functions. In 1885, Weierstrass gave a positive answer to this problem with his famous theorem, which states that every continuous function defined on a closed interval $[a, b]$ can be uniformly approximated by a sequence of polynomials. Since that time many researchers try to find an explicit form of such polynomials to give a simple proof of this theorem. A well-known and most celebrated proof of the Weierstrass approximation theorem for $f \in C[0,1]$ is due to Bernstein, in which he defined the following polynomials

$$
\left(B_{n} f\right)(x)=\sum_{k=0}^{n} f\left(\frac{k}{n}\right) p_{n, k}(x), \quad n \geq 1,
$$

This paper has been presented at the fourth edition of the International Conference on Numerical Analysis and Approximation Theory (NAAT 2018), Cluj-Napoca, Romania, September 6-9, 2018. 
where $p_{n, k}(x)=\left(\begin{array}{c}n \\ k\end{array}\right) x^{k}(1-x)^{n-k}$ is the Binomial distribution, and proved that $B_{n} f$ converges uniformly to any $f \in C[0,1]$ (see [7]). Further investigations are obtained by Lorentz in [19]. Since Bernstein operators are the prototype of many positive linear operators used in the theory of approximation, a great number of generalizations of these operators are given.

For the same functions, Stancu defined another positive linear operator as follows

$$
\left(P_{n}^{\alpha} f\right)(x)=\sum_{k=0}^{n} f\left(\frac{k}{n}\right) p_{n, k}^{\alpha}(x), \quad n \geq 1,
$$

where $\alpha$ is a non-negative parameter, which may depend only on the natural number $n$ and $p_{n, k}^{\alpha}(x)$ called Markov-Polya distribution (see [23]).

The special case $\alpha=0$ yields the Bernstein operator, while the Szasz-Mirakyan operator is shown to be a limiting case of $P_{n}^{\alpha}$. When $\alpha=1 / n$ we obtain the Lupas and Lupaş [20] operators corresponding to the equally spaced points $k / n(k=0,1, \ldots, n)$.

Up to the work of the famous polish mathematician Julian Musielak in 1981, see [22], the theory of approximation was strongly related with the linearity of the considered operators. Based on the idea developed in [22] and afterwards the works of C. Bardaro, G. Vinti and their research group on nonlinear operators, the approximation problem was proved by using nonlinear operators in some function spaces (see the fundamental book due to Bardaro, Musielak and Vinti [5]). For the approximation by linear and nonlinear operators, please see also the papers [3]-[2] and the monographs [10] and [26].

In view of the approach due to Musielak [22] and the techniques introduced by Bardaro-Mantellini in [4], Karsli-Tiryaki and Altin [18] considered the following nonlinear Bernstein operators;

$$
\left(N B_{n} f\right)(x)=\sum_{k=0}^{n} P_{n, k}\left(x, f\left(\frac{k}{n}\right)\right), 0 \leq x \leq 1, \quad n \in \mathbb{N},
$$

acting on bounded functions $f$ on an interval $[0,1]$, where $P_{n, k}$ satisfy some suitable assumptions. For further results we refer the papers [17], [16] and [18].

To generalize and extend the superposition or approximation problem for the functionals and operators, very recently in [13] and [14] Karsli defined and investigated the Urysohn type nonlinear Bernstein operators as;

$$
\left(N B_{n} F\right) x(t)=\int_{0}^{1}\left[\sum_{k=0}^{n} P_{k, n}\left(x(s), f\left(t, s, \frac{k}{n}\right)\right)\right] d s, 0 \leq x(s) \leq 1,
$$

where $P_{k, n}$ satisfy some suitable assumptions.

As a continuation of the above studies, in [14] the author also obtained Voronovskaya-type theorems for these operators.

For the linear forms of the Urysohn Bernstein and Urysohn Stancu operators we refer to the reader [11] and [21].

Moreover, in [15], Karsli considered a sequence $N B F=\left(N B_{n} F\right)$ of operators, which represents the Urysohn type nonlinear form of the two dimensional Bernstein 
operators defined by P.L. Butzer on the square $S=[0,1] \times[0,1]$ (see [8], [9]), having the form:

$$
\begin{aligned}
\left(N B_{n} F\right)(x(t), y(t))=\int_{0}^{1} \int_{0}^{1}[ & {\left[\sum_{k=0}^{n} \sum_{i=0}^{n} P_{k, i, n}\left(x(s), y(z), f\left(t, s, z, \frac{k}{n}, \frac{i}{n}\right)\right)\right] d s d z, } \\
& \leq x(s), y(z) \leq 1, n \in \mathbb{N},
\end{aligned}
$$

acting on bounded functions $f$ on $[0,1]^{5}$, where $P_{k, i, n}$ satisfy some suitable assumptions.

The central issue of this paper is to give a positive answer to the superposition problem for functionals and operators by introducing the Urysohn nonlinear operators of the two dimensional Stancu operators $\left(P_{n}^{\alpha} f\right)(x, y)$ defined on the triangle

$$
\triangle:=\{(s, z): s, z \geq 0, s+z \leq 1\} .
$$

Afterwards, we investigate the convergence problem for these nonlinear operators.

This paper is organized as follows: in Section 2, we construct the operators and further we present a basic lemma together with some definitions, which will be used in the sequel. Section 3 deals with the main convergence results for these operators.

\section{Preliminaries and auxiliary results}

This section is devoted to collecting some definitions and results which will be needed further on.

Now, we consider the following two dimensional Urysohn integral operator over the triangle $\triangle:=\{(s, z): s, z \geq 0, s+z \leq 1\}$,

$$
F(x(t), y(t))=\iint_{\triangle} f(t, s, z, x(s), y(z)) d s d z, \quad t, s, z \in[0,1]
$$

with unknown kernel $f$. If this representation exists, then $f(t, s, z, x(),. y()$.$) is called$ the two dimensional Green's function, which is strongly related to the functions $x$ and $y$ (see [25] and [26]).

In view of the above relations, we assume that the two dimensional continuous interpolation conditions hold:

$$
F\left(x_{i}(t), y_{j}(t)\right)=\iint_{\triangle} f\left(t, s, z, x_{i}(s), y_{j}(z)\right) d s d z, \quad t \in[0,1]
$$

where

$$
\begin{aligned}
x_{i}(s) & =\frac{i}{n} H(s-\xi) ; \xi \in[0 ; 1] \\
y_{j}(z) & =\frac{j}{n} H(z-\varsigma) ; \varsigma \in[0 ; 1]
\end{aligned}
$$


and $i, j=0,1,2, \ldots n$. By a straightforward calculation we have

$$
\begin{aligned}
\frac{\partial^{2} F\left(\frac{i}{n} H(s-\xi), \frac{j}{n} H(z-\varsigma)\right)}{\partial \xi \partial \varsigma}= & f\left(t, \xi, \varsigma, \frac{i}{n}, \frac{j}{n}\right)-f\left(t, \xi, \varsigma, \frac{i}{n}, 0\right) \\
& +f(t, \xi, \varsigma, 0,0)-f\left(t, \xi, \varsigma, 0, \frac{j}{n}\right)
\end{aligned}
$$

Say

$$
F_{1}\left(t, \xi, \varsigma, \frac{i}{n}, \frac{j}{n}\right):=\frac{\partial^{2} F\left(\frac{i}{n} H(s-\xi), \frac{j}{n} H(z-\varsigma)\right)}{\partial \xi \partial \varsigma} .
$$

According to the above definition, it is possible to construct an approximation operator in order to generalize and extend of the theory of interpolation of functions to operators.

For a bounded function defined on the triangle $\triangle:=\{(x, y): x, y \geq 0, x+y \leq 1\}$, two dimensional Stancu polynomials is given by:

$$
\left(P_{n}^{\alpha} f\right)(x, y)=\sum_{k=0}^{n} \sum_{j=0}^{n-k} p_{n, k, j}^{\alpha}(x, y) f\left(\frac{k}{n}, \frac{j}{n}\right),
$$

where $\alpha$ is a non-negative parameter, which may depend only on the natural number $n$ and

$$
\begin{aligned}
p_{n, k, j}^{\alpha}(x, y)= & \left(\begin{array}{l}
n \\
k
\end{array}\right)\left(\begin{array}{c}
n-k \\
j
\end{array}\right) \\
& * \frac{\prod_{l_{1}=0}^{k-1}\left(x+l_{1} \alpha\right) \prod_{l_{2}=0}^{j-1}\left(y+l_{2} \alpha\right) \prod_{l_{3}=0}^{n-k-j-1}\left(1-x-y+l_{3} \alpha\right)}{\prod_{l_{4}=0}^{n-1}\left(1+l_{4} \alpha\right)}
\end{aligned}
$$

is the two dimensional Markov-Polya distribution ([24]).

Finally, let us now consider a sequence $N P^{\alpha} F=\left(N P_{n}^{\alpha} F\right)$ of operators, which represents Urysohn type nonlinear counterpart of the two dimensional Stancu operators defined on the triangle $\triangle:=\{(s, z): s, z \geq 0, s+z \leq 1\}$, having the form:

$$
\begin{gathered}
\left(N P_{n}^{\alpha} F\right)(x(t), y(t)) \\
=\iint_{\triangle}\left[\sum_{k=0}^{n} \sum_{i=0}^{n-k} P_{k, i, n}^{\alpha}\left(x(s), y(z), f\left(t, s, z, \frac{k}{n}, \frac{i}{n}\right)\right)\right] d s d z, \\
0 \leq x(s), y(z) \text { and } x(s)+y(z) \leq 1, n \in \mathbb{N},
\end{gathered}
$$

acting on bounded functions $f$ on $[0,1]^{5}$, where $P_{k, i, n}^{\alpha}$ satisfy some suitable assump-

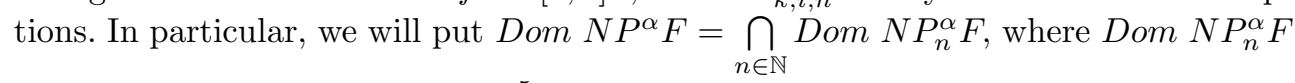
is the set of all functions $f:[0,1]^{5} \rightarrow \mathbb{R}$ for which the operator is well defined.

Let $X$ be the set of all bounded Lebesgue measurable functions

$$
f:[0,1]^{5} \rightarrow \mathbb{R}_{0}^{+}=[0, \infty) .
$$


Let $\Psi$ be the class of all functions $\psi: \mathbb{R}_{0}^{+} \rightarrow \mathbb{R}_{0}^{+}$such that the function $\psi$ is continuous and concave with $\psi(0)=0, \psi(u)>0$ for $u>0$.

We now introduce a sequence of functions. Let $\left\{P_{k, i, n}^{\alpha}\right\}_{n \in \mathbb{N}}$ be a sequence of functions $P_{k, i, n}^{\alpha}:[0,1] \mathrm{x}[0,1] \mathrm{x} \mathbb{R} \rightarrow \mathbb{R}$ defined by

$$
P_{k, i, n}^{\alpha}(t, l, u)=p_{k, n}^{\alpha}(t) p_{i, n}^{\alpha}(l) H_{n}(u)
$$

for every $t, l \in[0,1], u \in \mathbb{R}$, where $H_{n}: \mathbb{R} \rightarrow \mathbb{R}$ is such that $H_{n}(0)=0$ and $p_{k, n}^{\alpha}(\bullet)$ is the Markov-Polya basis.

Throughout the paper we assume that $\mu: \mathbb{N} \rightarrow \mathbb{R}^{+}$is an increasing and continuous function such that $\lim _{n \rightarrow \infty} \mu(n)=\infty$.

Assume that the following conditions hold:

a) $H_{n}: \mathbb{R} \rightarrow \mathbb{R}$ is such that

$$
\left|H_{n}(u)-H_{n}(v)\right| \leq \psi(|u-v|),
$$

holds for every $u, v \in \mathbb{R}$, for every $n \in \mathbb{N}$. That is, $H_{n}$ satisfies a $(L-\Psi)$ Lipschitz condition.

b) Denoting by $r_{n}(u):=H_{n}(u)-u, u \in \mathbb{R}$ and $n \in \mathbb{N}$, such that for $n$ sufficiently large

$$
\sup _{u}\left|r_{n}(u)\right|=\sup _{u}\left|H_{n}(u)-u\right| \leq \frac{1}{\mu(n)}
$$

holds.

The symbol $[a]$ will denote the greatest integer not greater than $a$.

Following our announced aim, in this part we recall results regarding the univariate and linear case of the celebrated Stancu operators.

Lemma 2.1. [23] For $\left(P_{n}^{\alpha} t^{s}\right)(x), s=0,1,2$, one has

$$
\begin{aligned}
\left(P_{n}^{\alpha} 1\right)(x) & =1 \\
\left(P_{n}^{\alpha} t\right)(x) & =x \\
\left(P_{n}^{\alpha} t^{2}\right)(x) & =x^{2}+\frac{(1+\alpha n) x(1-x)}{n(1+\alpha)} .
\end{aligned}
$$

By direct calculation, we find the following equalities:

$$
\left(P_{n}^{\alpha}(t-x)^{2}\right)(x)=\frac{x(1-x)(1+\alpha n)}{n(1+\alpha)},\left(P_{n}^{\alpha}(t-x)\right)(x)=0 .
$$

Moreover, for the second order central moment one has

$$
\left(P_{n}^{\alpha}(t-x)^{2}\right)(x) \leq \frac{1+\alpha n}{4 n(1+\alpha)} .
$$

Definition 2.2. Let $f \in C\left([a, b]^{5}\right)$ and $\delta>0$ be given. Then the complete modulus of continuity is given by:

$$
\omega(f ; \delta)=\sup _{\sqrt{\left(u_{1}-u_{2}\right)^{2}+\left(v_{1}-v_{2}\right)^{2}} \leq \delta}\left|f\left(t, s, z, u_{1}, v_{1}\right)-f\left(t, s, z, u_{2}, v_{2}\right)\right| .
$$


Further on, the partial modulus of continuity with respect to forth and fifth variables are defined by

$$
\omega_{1}(f ; \delta)=\sup _{t, s, z, v_{1}}\left(\sup _{\left|u_{1}-u_{2}\right| \leq \delta}\left|f\left(t, s, z, u_{1}, v_{1}\right)-f\left(t, s, z, u_{2}, v_{1}\right)\right|\right),
$$

and

$$
\omega_{2}(f ; \delta)=\sup _{t, s, z, u_{1}}\left(\sup _{\left|v_{1}-v_{2}\right| \leq \delta}\left|f\left(t, s, z, u_{1}, v_{1}\right)-f\left(t, s, z, u_{1}, v_{2}\right)\right|\right),
$$

respectively. Note that $\omega(f ; \delta)$ has the following properties;

(i) Let $\lambda \in \mathbb{R}^{+}$, then

$$
\omega(f ; \lambda \delta) \leq(\lambda+1) \omega(f ; \delta)
$$

(ii) $\lim _{\delta \rightarrow 0^{+}} \omega(f ; \delta)=0$,

(iii) $\left|f\left(t, s, z, u_{1}, v_{1}\right)-f\left(t, s, z, u_{2}, v_{2}\right)\right|$

$$
\leq \omega(f ; \delta)\left(1+\frac{\sqrt{\left(u_{1}-u_{2}\right)^{2}+\left(v_{1}-v_{2}\right)^{2}}}{\delta}\right) .
$$

The same properties also hold for partial moduli of continuity.

Now, we are ready to state some convergence results of the operators defined on the triangle.

\section{Main theorems}

Theorem 3.1. Let $F$ be the Urysohn integral operator with $0 \leq x(s), y(z)$ and

$$
x(s)+y(z) \leq 1 .
$$

Then $\left(N P_{n}^{\alpha} F\right)$ converges to $F$ uniformly in $x, y \in C[0,1]$. That is

$$
\lim _{n \rightarrow \infty}\left\|\left(N P_{n}^{\alpha} F\right)(x(t), y(t))-F(x(t), y(t))\right\|_{C(\triangle)}=0 .
$$

Proof. Owing to the definition of the operator given by (2.2), by considering (2.1), (2.3), (2.4) and (2.5), we have

$$
\begin{aligned}
& \left|\left(N P_{n}^{\alpha} F\right)(x(t), y(t))-F(x(t), y(t))\right| \\
= & \left|\iint_{\triangle}\left[\sum_{k=0}^{n} \sum_{i=0}^{n-k} P_{k, i, n}^{\alpha}\left(x(s), y(z), f\left(t, s, z, \frac{k}{n}, \frac{i}{n}\right)\right)\right] d s d z-F(x(t), y(t))\right|
\end{aligned}
$$




$$
\begin{aligned}
\leq & \iint_{\triangle} \sum_{k=0}^{n} \sum_{i=0}^{n-k} p_{k, n}^{\alpha}(x(s)) p_{i, n}^{\alpha}(y(z)) \cdot \\
& \cdot\left|H_{n}\left(f\left(t, s, z, \frac{k}{n}, \frac{i}{n}\right)\right)-H_{n}(f(t, s, z, x(s), y(z)))\right| d s d z \\
& +\iint_{\triangle} \sum_{k=0}^{n} \sum_{i=0}^{n-k} p_{k, n}^{\alpha}(x(s)) p_{i, n}^{\alpha}(y(z)) . \\
& \cdot\left|H_{n}(f(t, s, z, x(s), y(z)))-f(t, s, z, x(s), y(z))\right| d s d z \\
& :=I_{1}+I_{2} .
\end{aligned}
$$

Owing to the assumption $b$ ), one has

$$
\begin{aligned}
I_{2}= & \iint_{\triangle} \sum_{k=0}^{n} \sum_{i=0}^{n-k} p_{k, n}^{\alpha}(x(s)) p_{i, n}^{\alpha}(y(z)) \\
& \cdot\left|H_{n}(f(t, s, z, x(s), y(z)))-f(t, s, z, x(s), y(z))\right| d s d z \\
\leq & \iint_{\triangle} \sum_{k=0}^{n} \sum_{i=0}^{n-k} p_{k, n}^{\alpha}(x(s)) p_{i, n}^{\alpha}(y(z)) \frac{1}{\mu(n)} d s d z \\
= & \frac{1}{\mu(n)},
\end{aligned}
$$

which tends to zero as $n \rightarrow \infty$.

Using the definition of the function $F_{1}(t, s, z, x(s), y(z))$, by concavity of the function $\psi$, and using Jensen inequality, we obtain

$$
\begin{gathered}
I_{1} \leq \iint_{\triangle} \sum_{k=0}^{n} \sum_{i=0}^{n-k} p_{k, n}^{\alpha}(x(s)) p_{i, n}^{\alpha}(y(z)) \\
\times \psi\left(\left|f\left(t, s, z, \frac{k}{n}, \frac{i}{n}\right)-f(t, s, z, x(s), y(z))\right|\right) d s d z \\
\left.\leq \psi\left(\int \sum_{\triangle} \sum_{k=0}^{n} \sum_{i=0}^{n-k} p_{k, n}^{\alpha}(x(s)) p_{i, n}^{\alpha}(y(z))\left|f\left(t, s, z, \frac{k}{n}, \frac{i}{n}\right)-f(t, s, z, x(s), y(z))\right| d s d z\right)\right\} \\
\leq \psi\left\{\int_{\triangle} \sum_{k=0}^{n} \sum_{i=0}^{n-k} p_{k, n}^{\alpha}(x(s)) p_{i, n}^{\alpha}(y(z)) \times\left|F_{1}(t, s, z, x(s), y(z))-F_{1}\left(t, s, z, \frac{k}{n}, \frac{i}{n}\right)\right| d s d z\right. \\
\left.+\iint_{\triangle}\left|f(t, s, z, 0, y(z))-\sum_{i=0}^{n} p_{i, n}^{\alpha}(y(z)) f\left(t, s, z, 0, \frac{i}{n}\right)\right| d s d z\right\} \leq I_{1,1}+I_{1,2}+I_{1,3} .
\end{gathered}
$$


Let us divide the first term into four parts as;

$$
\begin{aligned}
I_{1,1} & =\psi\left(\begin{array}{c}
\iint_{\triangle} \sum_{k=0}^{n} \sum_{i=0}^{n-k} p_{k, n}^{\alpha}(x(s)) p_{i, n}^{\alpha}(y(z)) \cdot \\
\cdot\left|F_{1}(t, s, z, x(s), y(z))-F_{1}\left(t, s, z, \frac{k}{n}, \frac{i}{n}\right)\right| d s d z
\end{array}\right) \\
& : \quad \leq I_{1,1,1}+I_{1,1,2}+I_{1,1,3}+I_{1,1,4}
\end{aligned}
$$

where

$$
\begin{gathered}
I_{1,1,1}=\psi\left(\begin{array}{l}
\iint_{\triangle} \sum_{\left|\frac{k}{n}-x(s)\right|<\delta_{1}} \sum_{\left|\frac{i}{n}-y(z)\right|<\delta_{2}} p_{k, n}^{\alpha}(x(s)) p_{i, n}^{\alpha}(y(z)) \cdot \\
\cdot\left|F_{1}(t, s, z, x(s), y(z))-F_{1}\left(t, s, z, \frac{k}{n}, \frac{i}{n}\right)\right| d s d z
\end{array}\right), \\
I_{1,1,2}=\psi\left(\begin{array}{l}
\int_{\triangle} \sum_{\left|\frac{k}{n}-x(s)\right|<\delta_{1}} \sum_{\left|\frac{i}{n}-y(z)\right| \geq \delta_{2}} p_{k, n}^{\alpha}(x(s)) p_{i, n}^{\alpha}(y(z)) \cdot \\
\cdot\left|F_{1}(t, s, z, x(s), y(z))-F_{1}\left(t, s, z, \frac{k}{n}, \frac{i}{n}\right)\right| d s d z
\end{array}\right), \\
I_{1,1,3}=\psi\left(\begin{array}{l}
\iint_{\triangle}\left|\frac{k}{n}-x(s)\right| \geq \delta_{1}\left|\frac{i}{n}-y(z)\right|<\delta_{2} \\
\cdot\left|F_{1}(t, s, z, x(s), y(z))-F_{1}\left(t, s, z, \frac{k}{n}, \frac{i}{n}\right)\right| d s d z
\end{array}\right),
\end{gathered}
$$

and

$$
I_{1,1,4}=\psi\left(\begin{array}{l}
\iint_{\triangle} \sum_{\left|\frac{k}{n}-x(s)\right| \geq \delta_{1}} \sum_{\left|\frac{i}{n}-y(z)\right| \geq \delta_{2}} p_{k, n}^{\alpha}(x(s)) p_{i, n}^{\alpha}(y(z)) \cdot \\
\cdot\left|F_{1}(t, s, z, x(s), y(z))-F_{1}\left(t, s, z, \frac{k}{n}, \frac{i}{n}\right)\right| d s d z
\end{array}\right)
$$

Since $x, y \in C[0,1]$, then there exist $\delta_{1}, \delta_{2}>0$ such that

$$
\left|F_{1}(t, s, z, x(s), y(z))-F_{1}\left(t, s, z, \frac{k}{n}, \frac{i}{n}\right)\right|<\epsilon
$$

holds true when $\left|\frac{k}{n}-x(s)\right|<\delta_{1}$ and $\left|\frac{i}{n}-y(z)\right|<\delta_{2}$. So one can easily obtain

$$
I_{1,1,1}<\psi(\epsilon)
$$

As to the other terms

$$
\left|F_{1}(t, s, z, x(s), y(z))-F_{1}\left(t, s, z, \frac{k}{n}, \frac{i}{n}\right)\right| \leq 2 M
$$

holds true for some $M>0$, when $\left|\frac{k}{n}-x(s)\right| \geq \delta_{1}$ or $\left|\frac{i}{n}-y(z)\right| \geq \delta_{2}$. 
In view of Lemma 2.1, we obtain

$$
\begin{aligned}
& I_{1,1,2}=\psi\left(\begin{array}{c}
\iint_{\triangle} \sum_{\left|\frac{k}{n}-x(s)\right|<\delta_{1}} \sum_{\left|\frac{i}{n}-y(z)\right| \geq \delta_{2}} p_{k, n}^{\alpha}(x(s)) p_{i, n}^{\alpha}(y(z)) \cdot \\
\cdot\left|F_{1}(t, s, z, x(s), y(z))-F_{1}\left(t, s, z, \frac{k}{n}, \frac{i}{n}\right)\right| d s d z
\end{array}\right) \\
\leq & \psi\left(2 M \iint_{\triangle} \sum_{\left|\frac{k}{n}-x(s)\right|<\delta_{1}} \sum_{\left|\frac{i}{n}-y(z)\right| \geq \delta_{2}}\left(\frac{i-n y(z)}{\delta_{2}}\right)^{2} p_{k, n}^{\alpha}(x(s)) p_{i, n}^{\alpha}(y(z)) d s d z\right) \\
\leq & \left(2 M \iint_{\triangle} \sum_{\left|\frac{k}{n}-x(s)\right|<\delta_{1}\left|\frac{i}{n}-y(z)\right| \geq \delta_{2}}\left(\frac{i-n y(z)}{\delta_{2}}\right)^{2} p_{k, n}^{\alpha}(x(s)) p_{i, n}^{\alpha}(y(z)) d s d z\right) \\
\leq & \psi\left(\frac{2 M}{\delta_{2}^{2}} \frac{1+\alpha n}{4 n(1+\alpha)}\right) .
\end{aligned}
$$

Similarly one has

$$
I_{1,1,3} \leq \psi\left(\frac{2 M}{\delta^{2}} \frac{1+\alpha n}{4 n(1+\alpha)}\right)
$$

and

$$
I_{1,1,4} \leq \psi\left(\frac{2 M}{\delta_{1}^{2} \delta_{2}^{2}}\left(\frac{1+\alpha n}{4 n(1+\alpha)}\right)^{2}\right) .
$$

Collecting these estimates we have

$$
\begin{aligned}
& \left|\left(N P_{n}^{\alpha} F\right)(x(t), y(t))-F(x(t), y(t))\right| \\
\leq & \psi(\epsilon)+\psi\left(\frac{2 M}{\delta_{1}^{2}} \frac{1+\alpha n}{4 n(1+\alpha)}\right)+\psi\left(\frac{2 M}{\delta_{2}^{2}} \frac{1+\alpha n}{4 n(1+\alpha)}\right) \\
+ & \psi\left(\frac{2 M}{\delta_{1}^{2} \delta_{2}^{2}}\left(\frac{1+\alpha n}{4 n(1+\alpha)}\right)^{2}\right)+\frac{1}{\mu(n)} .
\end{aligned}
$$

That is

$$
\lim _{n \rightarrow \infty}\left\|\left(N P_{n}^{\alpha} F\right)(x(t), y(t))-F(x(t), y(t))\right\|_{C\left([0,1]^{2}\right)}=0 .
$$

This completes the proof.

Theorem 3.2. Let $F$ be the Urysohn integral operator with $x, y \in C[0,1]$ and $0 \leq x(s)$, $y(z) \leq 1$. Then

$$
\left|\left(N P_{n}^{\alpha} F\right)(x(t), y(t))-F(x(t), y(t))\right| \leq 2 \psi(\omega(f ; \delta))+\frac{1}{\mu(n)}
$$

holds true, where $\delta=\sqrt{\frac{1+\alpha n}{2 n(1+\alpha)}}$. 
Proof. Clearly one has

$$
\begin{aligned}
& \left|\left(N P_{n}^{\alpha} F\right)(x(t), y(t))-F(x(t), y(t))\right| \\
\leq & \iint_{\triangle} \sum_{k=0}^{n} \sum_{i=0}^{n-k} p_{k, n}^{\alpha}(x(s)) p_{i, n}^{\alpha}(y(z)) \cdot \\
& \cdot\left|H_{n}\left(f\left(t, s, z, \frac{k}{n}, \frac{i}{n}\right)\right)-H_{n}(f(t, s, z, x(s), y(z)))\right| d s d z \\
& +\frac{1}{\mu(n)} \\
:= & I_{n, 1}(x)+\frac{1}{\mu(n)},
\end{aligned}
$$

say. Since $x, y \in C[0,1]$ we can rewrite (3.1) as follows

$$
\begin{aligned}
& I_{n, 1}(x) \leq \iint \sum_{\Delta=0}^{n} \sum_{i=0}^{n-k} p_{k, n}^{\alpha}(x(s)) p_{i, n}^{\alpha}(y(z)) . \\
& \cdot \psi\left(\left|f\left(t, s, z, \frac{k}{n}, \frac{i}{n}\right)-f(t, s, z, x(s), y(z))\right|\right) d s d z \\
& \leq \iint_{\triangle} \sum_{k=0}^{n} \sum_{i=0}^{n-k} p_{k, n}^{\alpha}(x(s)) p_{i, n}^{\alpha}(y(z)) \psi(\omega(f ; \delta)) d s d z \\
& \leq \psi\left(\iint_{\triangle} \sum_{k=0}^{n} \sum_{i=0}^{n-k} p_{k, n}^{\alpha}(x(s)) p_{i, n}^{\alpha}(y(z)) \omega(f ; \delta) d s d z\right) \\
& \leq \psi\left(\begin{array}{c}
\iint_{\triangle} \sum_{k=0}^{n} \sum_{i=0}^{n-k} p_{k, n}^{\alpha}(x(s)) p_{i, n}^{\alpha}(y(z)) \cdot \\
\cdot\left(\frac{\sqrt{\left(\frac{k}{n}-x(s)\right)^{2}+\left(\frac{i}{n}-y(z)\right)^{2}}}{\delta}+1\right) \omega(f ; \delta) d s d z
\end{array}\right) \\
& =\psi\left(\begin{array}{c}
\omega(f ; \delta) \iint \sum_{\triangle}^{n} \sum_{k=0}^{n-k} p_{k, n}^{\alpha}(x(s)) p_{i, n}^{\alpha}(y(z)) \cdot \\
\cdot \frac{\sqrt{\left(\frac{k}{n}-x(s)\right)^{2}+\left(\frac{i}{n}-y(z)\right)^{2}}}{\delta} d s d z
\end{array}\right) \\
& +\psi\left(\omega(f ; \delta) \iint_{\triangle} \sum_{k=0}^{n} \sum_{i=0}^{n-k} p_{k, n}^{\alpha}(x(s)) p_{i, n}^{\alpha}(y(z)) d s d z\right) \\
& \leq \psi\left(\frac{\omega(f ; \delta)}{\delta} \iint_{\triangle}\left(\begin{array}{c}
\sum_{k=0}^{n} \sum_{i=0}^{n-k} p_{k, n}^{\alpha}(x(s)) p_{i, n}^{\alpha}(y(z)) \cdot \\
\cdot\left[\left(\frac{k}{n}-x(s)\right)^{2}+\left(\frac{i}{n}-y(z)\right)^{2}\right]
\end{array}\right)^{1 / 2} d s d z\right)+\psi(\omega(f ; \delta))
\end{aligned}
$$




$$
\leq \psi\left(\frac{\omega(f ; \delta)}{\delta}\left[\frac{1+\alpha n}{2 n(1+\alpha)}\right]^{1 / 2}\right)+\psi(\omega(f ; \delta)) .
$$

Taking into account that $\omega(f ; \delta)$ is the modulus of continuity defined as $(2.6)$. If we choose

$$
\delta=\sqrt{\frac{1+\alpha n}{2 n(1+\alpha)}}
$$

then one can obtain the desired estimate, namely,

$$
\left|\left(N P_{n}^{\alpha} F\right)(x(t), y(t))-F(x(t), y(t))\right| \leq 2 \psi(\omega(f ; \delta))+\frac{1}{\mu(n)} .
$$

Thus the proof is now complete.

Theorem 3.3. Let $F$ be the Urysohn integral operator with $x, y \in C[0,1]$, and $0 \leq x(s)$, $y(z) \leq 1$. Then

$$
\begin{gathered}
\left|\left(N P_{n}^{\alpha} F\right)(x(t), y(t))-F(x(t), y(t))\right| \\
\leq 2\left[\psi\left(\omega_{1}\left(f ;\left[\frac{1+\alpha n}{4 n(1+\alpha)}\right]^{1 / 2}\right)\right)+\psi\left(\omega_{2}\left(f ;\left[\frac{1+\alpha n}{4 n(1+\alpha)}\right]^{1 / 2}\right)\right)\right]+\frac{1}{\mu(n)}
\end{gathered}
$$

holds true.

Proof. In view of the definition of the considered operator, one has

$$
\begin{aligned}
& \left|\left(N P_{n}^{\alpha} F\right)(x(t), y(t))-F(x(t), y(t))\right| \\
& \leq \iint_{\triangle} \sum_{k=0}^{n} \sum_{i=0}^{n-k} p_{k, n}^{\alpha}(x(s)) p_{i, n}^{\alpha}(y(z)) \text {. } \\
& \cdot\left|H_{n}\left(f\left(t, s, z, \frac{k}{n}, \frac{i}{n}\right)\right)-H_{n}(f(t, s, z, x(s), y(z)))\right| d s d z \\
& +\frac{1}{\mu(n)} \\
& =\iint \sum_{\triangle} \sum_{k=0}^{n-k} p_{i=0}^{\alpha}(x(s)) p_{i, n}^{\alpha}(y(z)) \text {. } \\
& \cdot\left|\begin{array}{c}
H_{n}\left(f\left(t, s, z, \frac{k}{n}, \frac{i}{n}\right)\right)-H_{n}\left(f\left(t, s, z, x(s), \frac{i}{n}\right)\right) \\
+H_{n}\left(f\left(t, s, z, x(s), \frac{i}{n}\right)\right)-H_{n}(f(t, s, z, x(s), y(z)))
\end{array}\right| d s d z \\
& +\frac{1}{\mu(n)}
\end{aligned}
$$




$$
\begin{aligned}
& \leq \quad \iint_{\triangle} \sum_{k=0}^{n} \sum_{i=0}^{n-k} p_{k, n}^{\alpha}(x(s)) p_{i, n}^{\alpha}(y(z)) . \\
& .\left|H_{n}\left(f\left(t, s, z, \frac{k}{n}, \frac{i}{n}\right)\right)-H_{n}\left(f\left(t, s, z, x(s), \frac{i}{n}\right)\right)\right| d s d z \\
& \quad+\iint_{\triangle} \sum_{k=0}^{n} \sum_{i=0}^{n-k} p_{k, n}^{\alpha}(x(s)) p_{i, n}^{\alpha}(y(z)) \\
& \quad\left|H_{n}\left(f\left(t, s, z, x(s), \frac{i}{n}\right)\right)-H_{n}(f(t, s, z, x(s), y(z)))\right| d s d z \\
& \quad+\frac{1}{\mu(n)} \\
& =I_{n, 1}(x)+I_{n, 2}(x)+\frac{1}{\mu(n)},
\end{aligned}
$$

say. Since $x, y \in C[0,1]$ we can rewrite (3.1) as follows: By concavity of the function $\psi$, and using Jensen inequality, we obtain

$$
\begin{aligned}
I_{n, 1}(x)= & \iint_{\triangle} \sum_{k=0}^{n} \sum_{i=0}^{n-k} p_{k, n}^{\alpha}(x(s)) p_{i, n}^{\alpha}(y(z)) \cdot \\
& \cdot\left|H_{n}\left(f\left(t, s, z, \frac{k}{n}, \frac{i}{n}\right)\right)-H_{n}\left(f\left(t, s, z, x(s), \frac{i}{n}\right)\right)\right| d s d z \\
\leq & \iint \sum_{\triangle}^{n} \sum_{i=0}^{n-k} p_{k, n}^{\alpha}(x(s)) p_{i, n}^{\alpha}(y(z)) \psi\left(\omega_{1}\left(f ;\left|\frac{k}{n}-x(s)\right|\right)\right) d s d z \\
\leq & \psi\left(\iint_{\triangle} \sum_{k=0}^{n} \sum_{i=0}^{n-k} p_{k, n}^{\alpha}(x(s)) p_{i, n}^{\alpha}(y(z)) \omega_{1}\left(f ;\left|\frac{k}{n}-x(s)\right|\right) d s d z\right)
\end{aligned}
$$

Since $\psi$ is non decreasing, then one has

$$
\begin{aligned}
I_{n, 1}(x) \leq \psi\left(\begin{array}{c}
\iint \sum_{k=0}^{n} \sum_{i=0}^{n-k} p_{k, n}^{\alpha}(x(s)) p_{i, n}^{\alpha}(y(z)) \cdot \\
\cdot\left(\frac{\sqrt{\left(\frac{k}{n}-x(s)\right)^{2}}}{\delta_{1}}+1\right) \omega_{1}(f ; \delta) d s d z
\end{array}\right) \\
\leq \psi\left(\frac{\omega_{1}(f ; \delta)}{\delta}\left[\frac{1+\alpha n}{4 n(1+\alpha)}\right]^{1 / 2}\right)+\psi\left(\omega_{1}(f ; \delta)\right) .
\end{aligned}
$$

Similarly

$$
I_{n, 1}(x) \leq \psi\left(\frac{\omega_{2}(f ; \delta)}{\delta}\left[\frac{1+\alpha n}{4 n(1+\alpha)}\right]^{1 / 2}\right)+\psi\left(\omega_{2}(f ; \delta)\right)
$$


If we choose $\delta=\left[\frac{1+\alpha n}{4 n(1+\alpha)}\right]^{1 / 2}$, so we get the desired estimate.

Acknowledgments. The author is thankful to the referee for his/her valuable remarks and suggestions leading to a better presentation of this paper.

\section{References}

[1] Altomare, F., Campiti, M., Korovkin-Type Approximation Theory and its Applications, De Gruyter Studies in Mathematics, 17, Walter de Gruyter and Co., Berlin, 1994.

[2] Bardaro, C., Karsli, H., Vinti, G., Nonlinear integral operators with homogeneous kernels: pointwise approximation theorems, Appl. Anal., 90(2011), no 3-4, 463-474.

[3] Bardaro, C., Mantellini, I., On the reconstruction of functions by means of nonlinear discrete operators, J. Concr. Appl. Math., 1(2003), no. 4, 273-285.

[4] Bardaro, C., Mantellini, I., Approximation properties in abstract modular spaces for a class of general sampling-type operators, Appl. Anal., 85(2006), no. 4, 383-413.

[5] Bardaro, C., Musielak, J., Vinti, G., Nonlinear Integral Operators and Applications, De Gruyter Series in Nonlinear Analysis and Applications, Vol. 9, xii + 201 pp., 2003.

[6] Bardaro, C., Vinti, G., Urysohn integral operators with homogeneous kernel: approximation properties in modular spaces, Comment. Math. (Prace Mat.), 42(2002), no. 2, 145-182.

[7] Bernstein S.N., Demonstration du Théoreme de Weierstrass fondée sur le calcul des probabilités, Comm. Soc. Math. Kharkow, 13(1912/13), 1-2.

[8] Butzer, P.L., On Bernstein Polynomials, Ph.D. Thesis, University of Toronto, 1951.

[9] Butzer, P.L., On two dimensional Bernstein polynomials, Canad. J. Math., 5(1953), 107-113.

[10] Butzer, P.L., Nessel, R.J., Fourier Analysis and Approximation, Vol. 1, Academic Press, New York, London, 1971.

[11] Demkiv, I.I., On Approximation of the Urysohn operator by Bernstein type operator polynomials, Visn. L'viv. Univ., Ser. Prykl. Mat. Inform., (2000), no. 2, 26-30.

[12] Karsli, H., Approximation by Urysohn type Meyer-König and Zeller operators to Urysohn integral operators, Results Math., 72(2017), no. 3, 1571-1583.

[13] Karsli, H., Approximation Results for Urysohn Type Nonlinear Bernstein Operators, Advances in Summability and Approximation Theory, Book Chapter, Springer Nature Singapore Pte Ltd., 223-241, 2018.

[14] Karsli, H., Voronovskaya-type theorems for Urysohn type nonlinear Bernstein operators, Mathematical Methods in the Applied Sciences, (2018), accepted.

[15] Karsli, H., Approximation results for Urysohn type two dimensional nonlinear Bernstein operators, Const. Math. Anal., 1(2018), no. 1, 45-57.

[16] Karsli, H., Altin, H.E., A Voronovskaya-type theorem for a certain nonlinear Bernstein operators, Stud. Univ. Babeş-Bolyai Math., 60(2015), no. 2, 249-258.

[17] Karsli, H., Tiryaki, I.U., Altin, H.E., On convergence of certain nonlinear Bernstein operators, Filomat, 30(2016), no. 1, 141-155.

[18] Karsli, H., Tiryaki I.U., Altin H.E., Some approximation properties of a certain nonlinear Bernstein operators, Filomat, 28(6)(2014), 1295-1305.

[19] Lorentz, G.G., Bernstein Polynomials, University of Toronto Press, Toronto, 1953. 
[20] Lupaş, L., Lupaş, A., Polynomials of binomial type and approximation operators, Studia Univ. Babeş-Bolyai, Mathematica, 32(1987), 61-69.

[21] Makarov, V.L., Demkiv, I.I., Approximation of the Urysohn operator by operator polynomials of Stancu type, Ukrainian Math Journal, 64(2012), no. 3, 356-386.

[22] Musielak, J., On some approximation problems in modular spaces, In: Constructive Function Theory 1981, (Proc. Int. Conf., Varna, June 1-5, 1981), 455-461, Publ. House Bulgarian Acad. Sci., Sofia 1983.

[23] Stancu, D.D., Approximation of functions by a new class of linear polynomial operators, Rev. Roumaine Math. Pures Appl., 13(1968), 1173-1194.

[24] Stancu, D.D., A new class of uniform approximating polynomial operators in two and several variables, Proceedings of the Conference on the Constructive Theory of Functions (Approximation Theory) (Budapest, 1969), 443-455, Akademiai Kiado, Budapest, 1972.

[25] Urysohn, P., On a type of nonlinear integral equation, Mat. Sb., 31(1924), 236-255.

[26] Zabreiko, P.P., Koshelev, A.I., Krasnosel'skii, M.A., Mikhlin, S.G., Rakovscik, L.S., Stetsenko, V.Ja., Integral Equations: A Reference Text, Noordhoff Int. Publ., Leyden, 1975.

Harun Karsli

Bolu Abant Izzet Baysal University Faculty of Science and Arts

Department of Mathematics

14030 Golkoy Bolu, Turkey

e-mail: karsli_h@ibu.edu.tr 